\title{
Strain-Induced Crystallization I. Limiting Extents of Strain-Induced Nuclei*
}

\author{
G. S. Y. YEH \\ Departments of Chemical Engineering and \\ Materials \& Metallurgical Engineering and \\ Macromolecular Research Center \\ University of Michigan \\ Ann Arbor, Michigan
}

\begin{abstract}
This paper points out that interpretations from stress changes alone during oriented crystallization have led to widely different proposed chain conformations and consequently very different crystallization mechanisms for strain-induced crystallization (SIC). Many of the proposals, including the one by Keller and Machin which takes into account some electron microscopy and X-ray observations, show varying degrees of incompatibility with existing stress relaxation, kinetic or morphological data on SIC. Another problem lies in the difficulty with proper interpretation of observed morphology on samples which have been subjected to additional thermally-induced crystallization (TIC) after SIC, especially in the absence of prior characterization of SIC crystallites. The finding of a fibrillar-to-lamellar transformation in stretched polymers upon additional TIC (Part II) also indicates that the generally-observed oriented lamellar morphology has a much more subtle origin than that depicted by most crystallization models. Part I discusses our previously published morphological data on the characteristics of SIC crystallites from the melt, which includes: (a) their melting point elevation, $\left(T_{m} \gg T_{m}^{\circ}\right)$, (b) their nearly perfect crystalline orientation function $\left(f_{c} \sim 1\right),(c)$ their fast rates of crystallization $\left(t_{\frac{1}{2}}<1 \mathrm{sec}\right)$, and (d) their fibrillar morphology and limited dimensions along the fibrillar stretch axis $(\sim 100 \AA)$. Examples of morphology of SIC from the glass and from stirred solution are also included to show the overall similarity of fibrillar morphology brought about by stretching.
\end{abstract}

\section{INTRODUCTION}

C Currently there is a great deal of renewed interest in $\mathcal{C}_{\text {crystallization brought about by straining a polymer }}$ melt, solution or glass-the so-called stress- or strain-induced crystallization (SIC). This renewed interest is clearly indicated by the number of related papers on SIC at the 1975 March meeting of the American Physical Society, the American Chemical Society's Fiber and Yarn Processing Symposium in April, this SIC symposium (ACS, Chicago; August, 1975), and at two other symposia to be scheduled in the future (Budapest in 1976 and Anaheim, California in 1978). But the interest has always been there, since SIC is known to be a very important step during processing of fibers and films. When carried out under controlled conditions (1), SIC can induce morphologies that result in high strength, thermally more stable or optically less

* Previously presented at the ACS Symposium on Fiber and Yarn Processing, Philadelphia (April 9, 1975) opaque crystalline polymers. It is therefore important to get an accurate understanding of its morphology and kinetics of transformation, as well as its crystallization mechanism.

Starting as early as nearly half a century ago, Katz (2) was among the first to employ $\mathrm{X}$-ray diffraction to investigate crystallite orientation and size in "rubber-like (molten) synthetic substances which crystallize when stretched". About 30 years ago Flory (3) published the first thermodynamic treatment of crystallization of polymers induced by stretching, which included: a) the melting point elevation due to strain over and above the isotropic equilibrium melting point, and $b$ ) the relation between the increase in degree of crystallinity at constant elongation and temperature and the relative decrease in stress at crystallization equilibrium with elongation and temperature. The latter relation was derived on the assumption that crystallization (nucleation and growth) at constant elongation occurred along the strain direction, 
suggesting the following: a) that the strain-induced nuclei had a fringed-micelle chain conformation, and $b$ ) that the resultant crystallites due to SIC and/or additional thermally-induced crystallization (TIC) had dimensions comparatively greater in the strain than in the transverse directions. Such deductions about chain conformation and crystal dimensions from non-structural studies should be viewed with caution, especially if the deductions are based on an assumed crystal growth mechanism and direction that turned out to be wrong (4). Nevertheless, they seemed rather reasonable based on what one knew at the time about polymer morphology, especially if one assumed the starting amorphous polymer to have a random coil chain conformation. Stretching would be expected to extend and orient the randomly coiled macromolecules in the stretch direction. Upon phase transition, the aligned neighboring chain segments would be expected to lead directly to crystallites with a fringed-micelle chain conformation that is not so different from the as-stretched chain conformation. Any crystal growth would also be expected to continue along the stretch direction rather than perpendicular to it.

Flory's theoretical relations were qualitatively verified by Gent beginning in 1954 (5) in a series of experiments, including simultaneous volume and stress relaxation measurements from several polymers that were crystallized under strain at temperatures below their isotropic melting temperatures. The verification of Flory's theory also led Gent to conclude, which we quote from the Introduction of his 1966 paper (5), "the stress changes (decreases) in these cases (natural rubber and cis-polybutadiene) were in accord with the formation of oriented molecular crystallites and apparently incompatible with molecular folding, even at quite low extensions of the order of 15 percent. In addition, the crystal melting temperatures increased with the imposed extension in good agreement with Flory's treatment of oriented crystallization". The observed increase in axial stress by Gent during the latter stages of crystallization of trans-polyisoprene was attributed to volume contractions rather than to a different crystallization mechanism such as chain-folding, as later suggested by Keller and Machin (6).

Judge and Stein (7) and later Keller and Machin (6) reported axial length changes at constant load during crystallization of crosslinked polyethylene from the melt; the measured length always increased initially (approximately equivalent to stress decrease at constant strain), but contracted later, especially at small loads, and eventually leveled off at long times. Both Judge and Stein and Keller and Machin attributed the length change to a change in chain conformation from fringed-micelle (during length increase) to chain-folding (during contraction) during oriented crystallization. However, Judge and Stein suggested that the latter stages of crystallization occurred by chain-folding with a preferential $b$-axis alignment rather than with a preferential, experimentally observed, $c$-axis alignment in the stretching direction.
The observation of two distinct stages in the stress relaxation behavior was suggested by Keller and Machin as evidence in support of their proposed two-step nucleation and growth mechanism (6); the first stage began with strain-induced longitudinal nucleation of extended-chain crystals which they suggested to be occurring in the time periods, or up to $20-60 \mathrm{mins}$ in the case of polyethylene (8), during which the axial length was observed to increase, and the second stage followed with a thermally-induced lateral growth of chain-folded crystals which they suggested could explain the subsequent length contractions. They also suggested that the observed corresponding changes in crystal orientation from their wide angle X-ray scattering studies are in agreement with their proposed changes in crystallization mechanism during crystallization under strain.

In addition to the obvious departure (in the introduction of crystal growth by chain folding) from Flory's hypothetical model of oriented fringed-micelle crystallization, Keller and Machin's model has introduced two additional hypotheses. One is the extended-chain nucleus hypothesis which they claim is supported by measured melting point increases often associated with SIC. The other is the hypothesis that SIC can be readily differentiated from TIC during crystallization under strain at low temperatures by the changes seen in axial stress or axial length. We shall see that these two hypotheses are in conflict with a number of reported results based on morphological and kinetic studies done directly on SIC.

There is at present no direct evidence in support of the extended-chain hypothesis. Observed melting point increases due to SIC are neither direct evidence nor can they simply be construed as evidence for extended-chain crystals since the increases can be often as high as $100^{\circ} \mathrm{C}$ above the isotropic equilibrium melting point or the normal melting of extended-chain crystals. The melting point elevation is simply the result of reduction in melt entropy as has been correctly accounted for by Flory (3).

In contrast to the extended-chain hypothesis, morphological studies carried out directly on polymers crystallized by SIC (Part I) or on polymers after additional TIC (Part II) have inevitably led to the conclusions that SIC nuclei generally do not extend more than about $100 \AA$ in the stretch direction. Wide-angle X-ray studies, especially in the absence of detailed analysis, cannot provide unequivocal information on the extent of continuous crystal length. This was also pointed out by Hill and Keller (9) in the final paragraph of their paper, "Direct Evidence for Distinctive, Stress-Induced Nucleus in the Crystallization of Oriented Polymer Melts," which we quote, "So far nothing has been said about the nucleus morphology; the present X-ray information has nothing to add to this topic. It is only on the basis of the electron micrographs (Figs. 2-4 and others in Refs. [6, this paper] and [10, this paper]) and by analogy with the stirrer-induced crystallization that it is considered most probable that it is a fibrous morphology which provides a 
backbone on which lamellar crystals can grow in a direction transverse to the stress."

Although direct kinetic measurements of SIC are rare, available published data indicate that it generally completes within a fraction of a second for both natural rubber (11) and polyethylene $(12,13)$, much less than the 20 to 60 min over which the length was observed to increase or during which, according to Keller and Machin, the SIC process of polyethylene was supposed to be still continuing in the nucleation of extended-chain crystals. Even for a rigid molecule like poly-N-vinyl carbazole, crystallization is complete within one minute under strain (14). These kinetic results clearly indicate that the observed axial length increases (or stress decreases), in the time periods up to 20 to $60 \mathrm{mins}$, cannot be associated, as Keller and Machin had suggested, with the generation (or continuation) of extended-chain line nuclei during SIC but rather with the formation of perpendicularly-oriented chain-folded lamellae during TIC. Certainly this seems a most surprising and probably somewhat unbelievable conclusion if not for the fact that there are other examples which lead to the same conclusion.

For example, we have, through a series of comprehensive electron microscopy (15), small- and wideangle X-ray (16) and stress-relaxation (4) experiments, established that perpendicularly-oriented chain-folded lamellae form in stretched natural rubber upon further supercooling (TIC) to below about $18^{\circ} \mathrm{C}$, the same temperature region in which complete stress relaxation (stress is never observed to increase in natural rubber) is normally obtained $(4,5)$. Indeed, the formation of chain-folded lamellae with their $c$-axis parallel to the stretch direction during stress decay may seem like such a physical impossibility that it was probably never even considered by Andrews in his natural rubber studies (17). He proposed a fringed-micelle crystallization mechanism not only for his so-called $\gamma$ type crystalline filaments (parallel to the stretch direction) but also for his $\alpha$ type crystalline filaments (perpendicular to the stretch direction) (17). The formation of chain-folded lamellae in stretched natural rubber during stress decrease is undoubtedly another experimental fact unaccounted for by Keller and Machin's model since their model should predict a stress increase or at least an eventual upturn in stress. However, the predicted upturn is never observed in natural rubber $(4,5)$.

It is of interest to note that, in spite of these widely different and erroneous deductions from stress relaxation studies about chain conformation and crystallization mechanism of SIC, the explanation we had given for the stress relaxation during the formation of chain-folded lamellae remained basically the same as that offered by Flory. We showed (4) that crystallization from oriented chain segments, but with numerous prefolds, should lead to stress decrease under constant strain because the stress decrease is primarily brought about by the greater conformational freedom given to the "connecting chain segments" in the noncrystalline regions either during the nucleation of strain induced crystallites (Part I) or during the fibrillar-to-lamellar transformation upon TIC
(Part II). However, the number of "connecting chain segments" must be fewer than that generally pictured in the classical fringed-micelle type crystal conformation; otherwise, crystal growth during SIC and/or TIC would be expected to continue along the stretch direction, but this is never observed experimentally (Part II).

Aside from this kind of interpretive problem that is generally associated with the use of non-structural techniques in assessing crystal morphology and chain conformation, there is another kind of interpretive problem in many of the reported morphological studies. It usually arises from using samples that have been subjected to substantial additional crystallization at low temperatures (TIC) after SIC. The use of such preparations has two major drawbacks. First, it eliminates the opportunity for direct detailed studies of strain-induced nucleus morphology necessary for deducing SIC crystallization mechanism(s). Second, there is an inherent danger in simply assuming the observed morphology to come directly from a melt, oriented or partially relaxed, without any intermediate steps. For example, oriented lamellae have often been pictured to crystallize directly from a relaxed melt by epitaxy onto some line nuclei as in the model by Keller and Machin. The experimental evidence discussed in Part II, which shows how various starting crystalline or noncrystalline fibrillar morphologies can lead to more or less similar oriented lamellar morphology, clearly raises the validity of such a simple assumption.

Similarly, studies of SIC morphology without extensive follow-up studies of the morphology due to additional TIC may lead to erroneous deductions about the final morphology. An example is seen in the natural rubber studies by Andrews (17) where he ruled out any possibility of lamellar formation in highly stretched natural rubber at lower temperatures (TIC), simply because the as-stretched sample is already full of fibrillar crystals. He did not realize that lamellae could develop with time at low temperatures, as established from both electron microscopy (15) and small-angle X-ray scattering evidence (16).

This paper is divided into two parts in order to emphasize the difference between SIC morphology and the morphology of SIC after additional TIC. Part I deals with the reported morphology of crystals brought about solely by the straining of polymer melts, glasses or solutions; i.e., in the absence of strain, such SIC crystals will not normally form at the given temperature. The SIC crystals are also readily identifiable by their higher melting point, often higher than that of the extendedchain crystals. Part II deals with a reported morphological transformation which occurs upon additional TIC of strain-crystallized polymers. The typical examples cited in Parts I and II are mostly taken from various SIC studies which we started first in 1962 with natural rubber and which are still being continued today with other polymers. Most of the cited results are taken from our thin film studies which, in conjunction with our bulk studies, have played a decisive role in our conclusions to date on the SIC morphology and on the morphology developed upon further TIC. 


\section{STRAIN-INDUCED CRYSTALLIZATION OF POLYMER IN OR NEAR THE RUBBERY MOLTEN STATE}

We have reported morphological studies on the SIC of natural rubber (cis-polyisoprene) $(15,16)$ and gutta percha (trans-polyisoprene) (18, 19), both by elongational straining, and Marlex 6050 polyethylene by shear (1). Natural rubber is found to be the most ideal as far as elucidation of the SIC nucleus morphology is concerned. It has an isotropic melting point of about $25^{\circ} \mathrm{C}$, and because of its high molecular weight (in the millions), it can easily undergo SIC at the convenient room temperature either in its uncrosslinked or crosslinked molten state. Because it also crystallizes very slowly in the absence of straih and has a limiting crystallinity of less than 40 percent either by stretching and/or additional TIC (20), the SIC crystals can be readily identified and differentiated from the TIC crystals, e.g., in kinetic studies. In contrast, polyethylene has none of the advantages that natural rubber has. Marlex 6050 polyethylene crystallizes very fast in the absence of strain. It cannot be made to crystallize solely by SIC without additional TIC. Consequently, we encountered some difficulty in separating one type of crystals (SIC) from another (TIC) in the case of polyethylene.

One major conclusion that came out of these three studies was that SIC always tends to generate a fibrillar morphology in the absence of TIC regardless of the applied strain as long as the polymer has reached the critical strain for SIC at a given temperature. This is in contrast to the general contention that the morphology of SIC changes from lamellar for samples with low applied strains to fibrillar for samples with high applied strains. We shall see in Part II that changes in morphology can take place if the stretched (or SIC) material has been subjected to additional thermal treatment (or TIC).

Another major finding is that the fibrillar morphology consists of fibrils which have diameters of about $100 \AA$ and contain SIC crystallites with limiting crystal thicknesses. They range from about $150 \AA$ for natural rubber and $250 \AA$ for gutta percha to about $200-400 \AA$ for polyethylene in the strain direction. The following are some typical examples taken from our natural rubber studies.

When natural rubber is stretched above its critical strain for SIC or about 250 percent at room temperature, it crystallizes almost instantly with a typical fibrillar morphology. This is shown in Fig. 1 for a 300 percent strained and in Fig. 2 for a 700 percent strained thin film. The crystal dimensions within the fibrils, as determined directly from the extent of coherent scattering regions from the dark field micrograph (Fig. 1, left) or from the extent of unstained regions (Fig. 1, right and Fig. 2), vary widely between about $50 \AA$ and $150 \AA$ in both the longitudinal and the lateral directions to the fibril or stretch axis and give rise to a particulate fibrillar morphology. Such particulate fibrils can be seen to be present throughout the more highly strained samples (Fig. 2). Heating above room temperatures or to 45 and $75^{\circ} \mathrm{C}$ (15) showed that they have high elevated melting points,

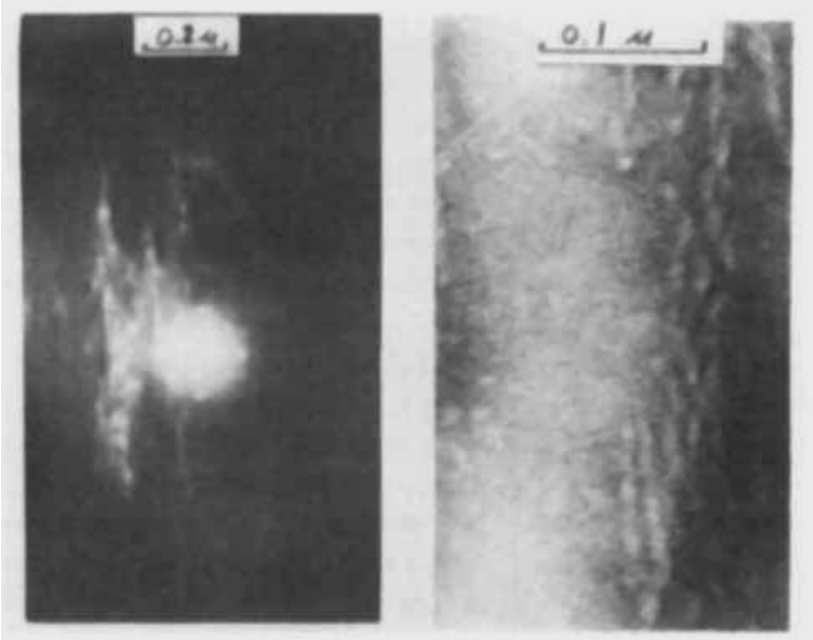

Fig. 1, Natural rubber stretched 300 percent. Left: dark field with superimposed electron diffraction. Right: $\mathrm{OsO}_{4}$ stained (15).

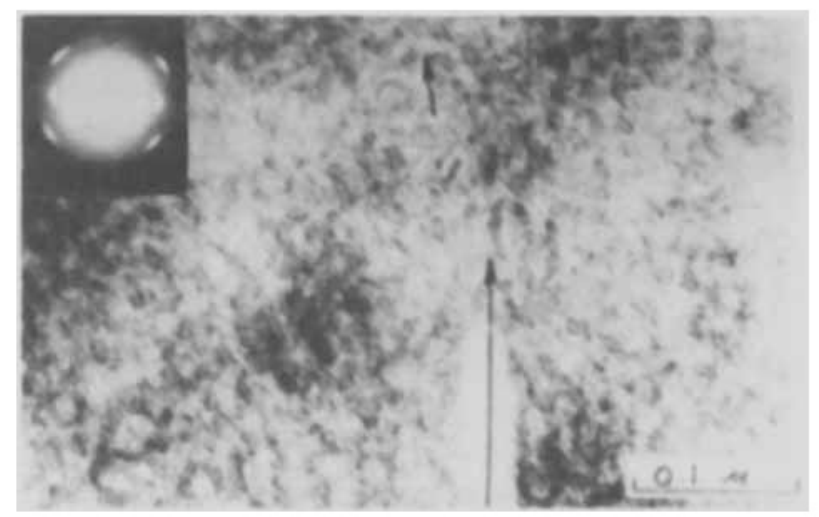

Fig. 2. Natural rubber stretched 700 percent after aging at room temperature for 5 months and then $\mathrm{OsO}_{4}$ stained; the morphol ogy remains almost the same as freshly stretched to 700 percent (15).

as expected of SIC crystallites. Furthermore, it was noted that although the number of fibrils decreased with increasing temperature, no apparent change was observed in the remaining particulate fibrils. Consequently, the possibility that the observed particulate structures were due to some type of chain-folded crystal overgrowth as a result of normal supercooling effects was ruled out.

Two other points are worth mentioning. One is their near perfect crystal orientation as judged by their corresponding electron diffraction (Fig. 1, left and Fig. 2); extremely high $c$-axis orientation functions ranging from 0.95 to 0.99 for applied strains between 250 and 650 percent have been reported by Kawai $e t$ al. (21). The other concerns the noticeable tendency for SIC crystallites in neighboring fibrils to align laterally with one another, especially after some stress relaxation at room temperature after 5 months (see Fig. 2).

\section{STRAIN-INDUCED CRYSTALLIZATION OF POLYMERS IN THE GLASSY STATE}

In a strict sense, strain-induced crystallization does not occur in the glassy state because of the lack of atomic mobility necessary for atomic rearrangement into a 
3-dimensional crystalline lattice. For some polymers, such as polyethylene terephthalate (PETP) and polycarbonate (PC), a noticeable improvement in atomic packing can be brought about by stretching in their glassy state. An example will be given later. For others like isotactic polystyrene (IPS) or plasticized IPS, which we have studied (22), stretching simply brings about some molecular and fibrillar orientation along the direction of stretch.

Regardless of the extent of improvement in atomic packing, the extent of molecular orientation was found to be much higher than that normally obtainable by stretching in the melt, as expected, because of lack of extensive molecular relaxation in the glassy state. Therefore, we were very much interested in the molecular chain conformation in the resultant crystals brought about by additional TIC from essentially noncrystalline but highly oriented molecules. Examples of their morphology will be given in Part II.

Two readily detectable changes show up immediately in all polymers when sufficiently stretched in the glassy state. For example, the diffraction pattern and its corresponding area of a 500 percent stretched PETP (Fig. 3, top) tell us immediately that excellent molecular alignment and oriented fibrils can be achieved parallel to the stretch direction. Because of the molecular anisotropy introduced by the stretching process, such stretched films also tend to split easily along the stretch direction.

There are other features which are much less obvious and require more careful and detailed studies. For example, the diffraction pattern of PETP shows that the

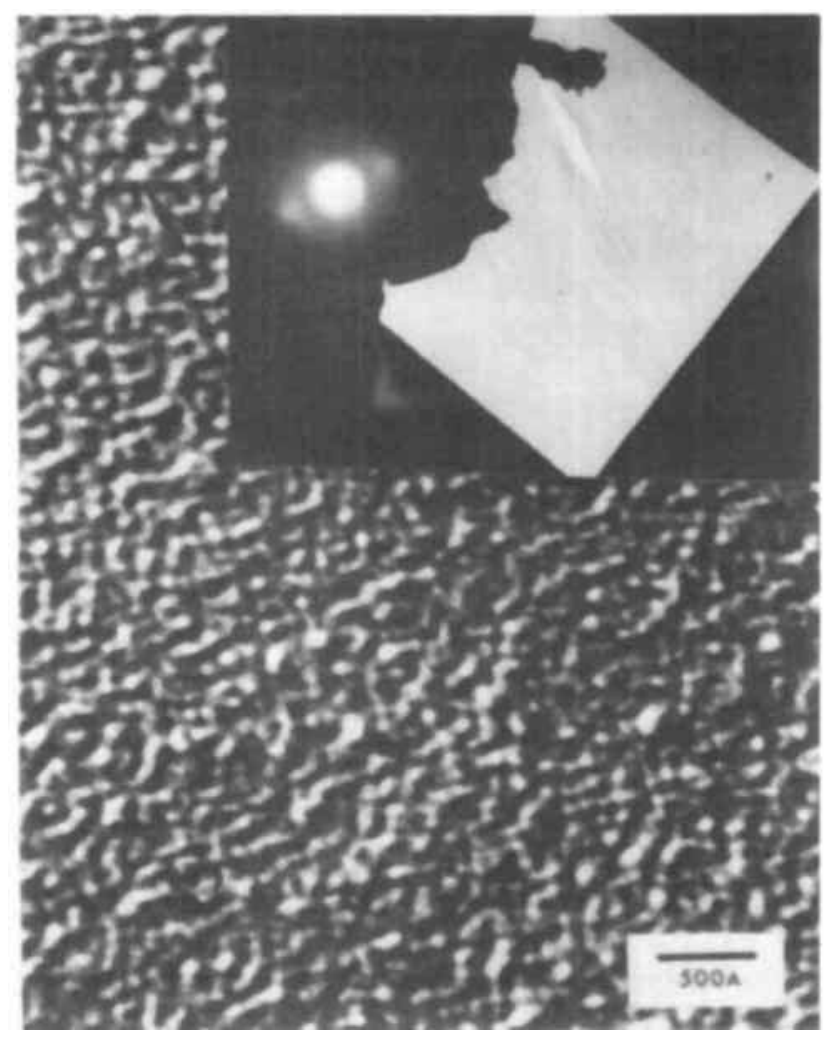

Fig. 3. PETP stretched 500 percent at $65^{\circ} \mathrm{C}, \mathrm{Pt}$-shadowed. The insert shows an electron diffraction pattern and its corresponding unshadowed area (23). meridional reflection peaks are much sharper (best seen on the original negative) than the diffuse peaks along the equator (Fig. 3, top). This was also noted in the wideangle X-ray patterns of bulk stretched PETP and could be explained by the presence of smectic packing of chain segments as a result of the strain (23). Similarly, if one examined the morphology more carefully under higher resolutions, one can detect structures ranging between 50 and $100 \AA$ and aligning more or less at some angle (along the maximum shear) to the stretch direction (Fig. 3 ). The arrangement of these structures was also reflected in the small-angle $\mathrm{X}$-ray scattering from bulk stretched PETP, which showed detectable but somewhat diffuse and faint 4-point patterns (23). The presence of small-angle scattering maxima, with corresponding periodicities limited to $50-100 \AA$, clearly indicates that the extent of order (or denser regions) introduced by stretching in the stretch direction must also be limited to less than $100 \AA$ or to about the size of structures seen in the electron micrograph.

From the examples given so far, one can conclude that, while there are distinct differences between SIC in the melt and strain-induced "crystallization" in the glass, there are some very interesting similarities between the two, namely the presence of a fibrous texture, good $c$-axis alignment and in some cases some limited extent of crystalline or smectic order along the stretch direction, whenever a polymer is sufficiently stretched in the melt or in the glass.

\section{SHEAR-INDUCED CRYSTALLIZATION IN THE SOLUTION STATE}

As others before us, we were also very much intrigued by the so-called shish-kebab structures that were first observed by Pennings and Kiel (24) in stirred polyethylene solutions. In particular, we were most interested in the structure of the central shish, presumably resulting from the stirring action.

Ideally, if individual crystals can be generated by shearing at the dissolution temperature, they would be even more suitable for SIC studies than those generated from the melt at the melting temperature. Unfortunately, we soon learned that pure shishes, presumably the SIC nuclei, were extremely difficult, if not totally impossible, to produce at the dissolution temperature (25). Even with repeated careful washings with fresh xylene at the highest possible crystallization temperatures (i.e., the smallest supercoolings) and quick quenching to low temperatures, etc., the "cleanest" shishes still consist of some microkebabs (Fig. 4, right). It was not until after numerous dark field and deformation experiments (25) that we began to realize that the microkebabs were most likely an integral part of the shish and permanently attached to it by tie molecules in a manner as shown in the inserted schematic drawing (Fig. 4). The evidence for the limited crystal thickness $(200 \AA)$ depicted in the drawing stems primarily from dark field microscopy and gold decoration studies (25), but also partly from the ductile failure modes which they tend to exhibit when they are stretched individually (Fig. 5). (A brittle type of failure mode would be ex- 


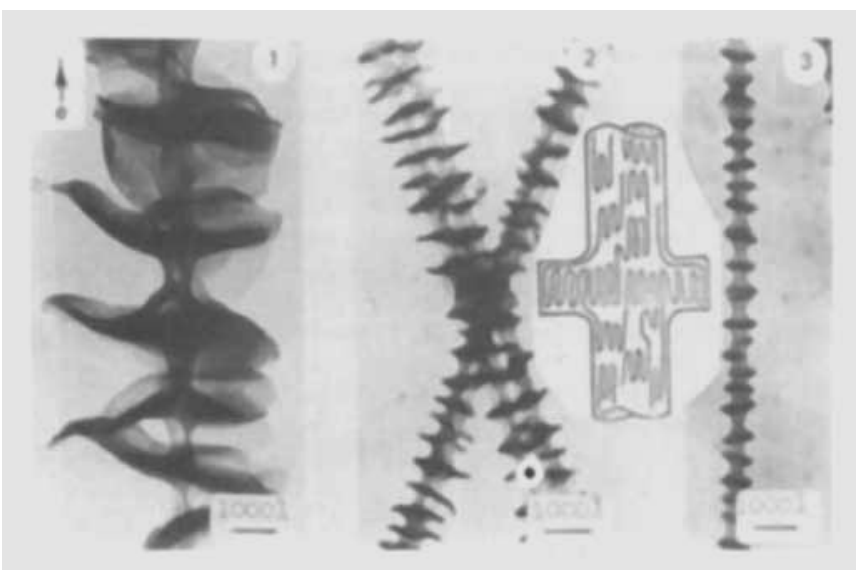

Figure 4. Stirrer-crystallized polyethylene at $100^{\circ} \mathrm{C}$ with different washing and quenching conditions (25). The insert shows a schematic drawing of the chain conformation for the "cleanest" shish-kebabs.

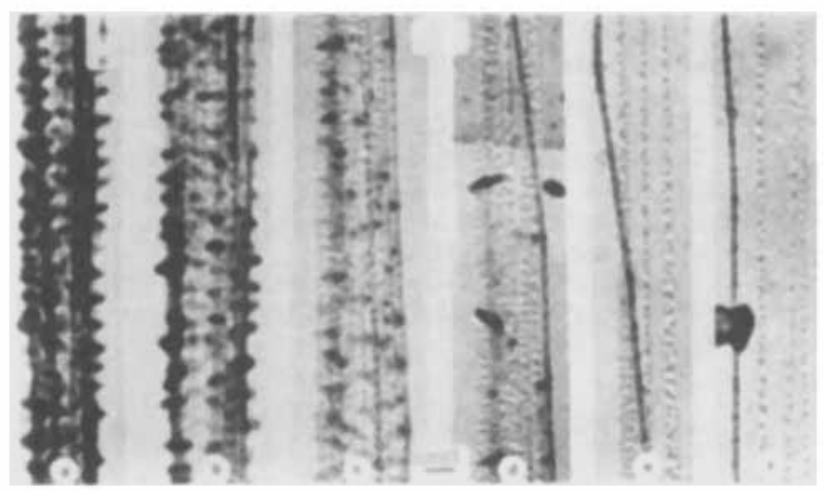

Fig. 5. Series of successive micrographs of a drawn bundle of gold decorated shish-kebab fibers: (a) undrawn region, $(b),(c)$, (d) transition regions and $(e),(f)$ highly drawn region (25).

pected if the shishes are made of extended-chain crystals. The reportedly observed brittle failure in a macroscopic "bulk" test of a packed bundle of shish-kebabs cannot be directly construed as evidence for extendedchain crystals because of non-uniformity in packing.) The gradual disappearance of the microkebabs seen in Fig. 5 also tends to support the suggested composite nature of such shish-kebabs.

It is believed the origin of microkebabs is probably the same as those seen in strain-crystallized natural rubber (Fig. 2) where the SIC crystallites are seen tending to pack laterally, the primary driving force being the lowering of total crystal surface free energy. Another similarity when compared with SIC of natural rubber is found in their melting point elevation. These polyethylene microshish-kebabs often show a high temperature tail in our DSC scans that extends up to $150-152^{\circ} \mathrm{C}$. Pennings et al. (26) made the same observation in 1970. However, they attributed the melting point elevation to the presence of an extended-chain crystalline backbone.

\section{LIMFTING STRAIN-INDUCED NUCLEUS THICKNESS}

The results discussed above have come from a wide variety of SIC studies which all seem to indicate that SIC tends to result in a fibrillar morphology, a high degree of $c$-axis orientation and a melting point elevation. These facts are more or less well-known, expected and generally accepted. A rather unexpected finding from all our studies is the size of SIC crystals, limited to about $100 \AA$ in the fibrillar or the stretch direction. Although the finding comes mainly from our studies, we should point out that measurements by others from line broadening (27) and small-angle X-ray peak positions $(12,13)$ also indicate the size of SIC nuclei to be limited to about

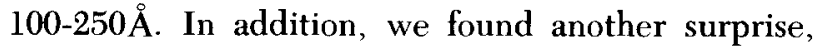
namely that the subsequent crystal growth did not proceed along the fibrils or along the aligned molecules, as one would expect upon further TIC, but perpendicular to the already aligned molecules (Part II). Our studies on the amorphous state of polymers were originally initiated for the purpose of understanding our findings in SIC studies. Although we had shown that there appears a direct correlation between size of structures established in the melt or the glass (28) and the size of straininduced crystallites, there may be other contributing factors which need to be considered also.

From thermodynamics of crystallization, regardless of folded or fringed-micelle crystallization mechanism, one can show that the thickness of critical nucleus is $1_{c}=$ $4 \sigma_{e} / \Delta f$, where $\sigma_{e}$ and $\Delta f$ are the end surface free energy of the crystal and the difference in free energy of fusion between the melt and the crystal at a given crystallization temperature. The crystal nuclei induced by straining should be smaller than that formed in the isotropic state if $\sigma_{e}^{\circ}$ for the strained case remains the same as $\sigma_{e}$ for the unstrained case. This was pointed out by Kobayashi and Nagasawa (29) because $(\Delta f)^{\circ}$ for the oriented crystallization case should be larger than $\Delta f$ for the unoriented due to the reduction in melt entropy, which results from a reduction in the difference in entropy, $\Delta S$, between the oriented melt and the crystal and therefore a larger $(\Delta f)^{\circ}$. Even if we take into account the straininduced crystallization rate increases, which suggest (30) $\sigma_{e}^{\circ}$ may range between $\sigma_{e} / 2$ and $2 \sigma_{e}(30)$, the size of critical strain-induced nuclei should still be in the order of $100 \AA$, since $(\Delta f)^{\circ}$ should always be larger than $\Delta f$.

Another origin may have to do with the limited degree of chain extensions that may be induced in the molten or solution state. Peterlin's estimate of the maximum possible deformation of a random coil, under the assumption of pure extensional flow and without any thermal relaxation (e.g., in the glassy state), indicates (31) that the fraction of fully extended chain segments is very small, on the order of about 1 percent for most polymers at a macroscopic draw ratio of 5 and molecular weight of 30,000. Direct birefringence measurements by Mackley and Keller (32) of molten polyethylene during elongational flow at $140^{\circ} \mathrm{C}$ indicate values to be about 2.5 times below the theoretical value for fully aligned molecules. Our own calculations from analysis of measured rate increase of shear-induced crystallization of polyethylene (1) indicate that the average molecular chain extension in the melt just prior to crystallization is only about 50 percent (30).

All the findings, theoretical and experimental, suggest that the SIC nuclei have limited dimensions along the stretch direction. These results may explain 
why extended-chain crystals are very difficult, if not totally impossible, to produce by SIC alone, as for example was noticed in the case of impinging jet experiments (33).

\section{ACKNOWLEDGMENT}

The author wishes to take this opportunity to thank the Fulbright Commission for a Senior Research Fellowship award. The author is also grateful to the National Science Foundation for their partial support of the research.

\section{REFERENCES}

1. D. Krueger and G. S. Y. Yeh, J. Appl. Phys., 43, 4339 (1972).

2. J. R. Katz, Naturw., 4, 169 (1925) and Trans. Faraday Soc., 32, 77 (1936).

3. P. J. Flory, J. Chem. Phys., 15, 397 (1947).

4. D. Luch and G. S. Y. Yeh, J. Polym. Sci., 11, 467 (1973).

5. A. N. Gent, Trans. Faraday Soc, 50, 521 (1954); J. Polym. Sci., A3, 3787 (1965); ibid, A2, 4, 447 (1966).

6. A. Keller and M. J. Machin, J. Macromol. Sci.(Phys.), B1, 41 (1967).

7. J. T. Judge and R. S. Stein, J. Appl. Phys., 32, 2357 (1962).

8. M. J. Hill and a Keller, J. Macromol. Sci. (Phys.), B5, 591 (1971).

9. M. J. Hill and A. Keller, J. Macromol. Sci. (Phys.), B3, 153 (1969).

10. M. J. Machin, Ph.D. Thesis, Bristol University, 1967.

11. J. C. Mitchell and D. J. Meier, J. Polym. Sci., A2, 6, 1689 (1968).

12. K. Katayama, T. Amano, and K. Nakamura, Kolloid-Z Polymere, 226, 125 (1968).
13. J. R. Dees and J. E. Spruiell, J. Appl. Polym. Sci., 18, 1053 (1974).

14. T. K. Su, Ph.D. Thesis, Univ. of Massachusetts, 1975.

15. D. Luch and G. S. Y. Yeh, J. Appl. Phys., 43, 4326 (1972).

16. D. Luch and G. S. Y. Yeh, J. Macromol. Sci. (Phys.), B7, 121 (1973).

17. E. H. Andrews, Proc. Royal Soc., A277, 562 (1964).

18. I. R. Hardin and G. S. Y. Yeh, J. Macromol. Sci. (Phys.), B7, 375 (1973).

19. I. R. Hardin and G. S. Y. Yeh, J. Macromol. Sci.(Phys.), B7, 393 (1973).

20. G. S. Y. Yeh, J. Macromol. Sci. (Phys.), B8, 241 (1973).

21. H. Hiratsuka, M. Hashiyama, S. Tomita, and H. Kawai, Paper presented at U.S.-Japan Joint Seminar, Cleveland, Oct. 9-13 (1972).

22. G. S. Y. Yeh and S. L. Lambert, J. Appl. Phys., 42, 4614 (1971).

23. G. S. Y. Yeh and P. H. Geil, J. Macromol. Sci. (Phys.), B1, 251 (1967).

24. A. J. Pennings and A. M. Kiel, Kolloid-Z Polymere, 205, 16 (1965).

25. K. Krueger and G. S. Y. Yeh, J. Macromol. Sci. (Phys.), B6, $431(1972)$.

26. A. J. Pennings, J. M. A. A. van der Mark and A. M. Kiel, Kolloid-Z Polymere, 237, 336 (1970).

27. E. H. Andrews, J. Polym. Sci., A4, 66 (1966).

28. G. S. Y. Yeh, J. Macromol. Sci. (Phys.), B6, 465 (1972).

29. K. Kobayashi and T. Nagasawa, J. Macromol. Sci.(Phys.), B4, 331 (1970).

30. K. Z. Hong and G. S. Y. Yeh, to be published.

31. A. Peterlin, Polym. Eng. Sci., 14, 627 (1974).

32. M. R. Mackley and A. Keller, Polymer, 14, 16 (1973).

33. F. C. Frank, A. Keller and M. R. Mackley, Polymer, 12, 467 (1971). 